\title{
Discontinuous dynamical systems and synchronization
}

\author{
Albert C.J. Luo ${ }^{\mathrm{a}}$ \\ Department of Mechanical Engineering, Southern Illinois University Edwardsville, Edwardsville, \\ IL 62026-1805, USA
}

Received 13 May 2019

Published online 30 July 2019

This special issue is on discontinuous dynamical systems and synchronization. Discontinuous dynamical systems exist extensively in physical systems, which are also used for control in engineering. For such discontinuous dynamical systems, the traditional analysis cannot provide a better understanding of the corresponding dynamics. Thus, the studies of discontinuous dynamical systems become very significant. In 1950s, one started to investigate such problems, and the sliding mode control was developed. However, the mathematics theory and physical mechanisms of discontinuous dynamical systems should be investigated comprehensively. From the recent development of discontinuous dynamical systems, engineering discontinuous dynamical systems have been investigated and dynamical system synchronization has been studied. This special issue collects such recent results together. Objectives of this special issue are:

- to present the recent developed theory and methods for discontinuous dynamical systems,

- to apply discontinuous dynamical system theory to engineering problems,

- to predict periodic motions and chaos in discontinuous dynamical systems

- to help readers understand the complexity of periodic motions in discontinuous dynamical systems.

To achieve the aforementioned objectives, four topics are: (i) the discontinuous dynamical systems with switching at the boundary; (ii) the impact dynamics in Fermi-oscillator, impact dampers and energy harvesting systems; (iii) the synchronization of dynamical systems, (iv) infinite-equilibrium systems. The four topics are distributed through the following papers.

- Complexity of dynamic system switching between two subsystems with cornered boundaries [1]

- Bifurcation and chaos of a periodically forced Duffing system with absolute function [2]

- Acceleration behaviors of fermi accelerator model excited by van der Pol Oscillator [3]

- Stability and bifurcation of periodic motions in an inclined impact pair [4]

\footnotetext{
a e-mail: albert.cj.luo@gmail.com
} 
- Energy harvesting of a frequency up-conversion piezoelectric harvester with controlled impact [5]

- Piezoelectric frequency up-conversion harvester under sawtooth wave excitation $[6]$

- Chaos synchronization of three distinct non-autonomous system with periodic excitation [7]

- Synchronization in flow around airfoil and local oscillating flexible surface at high angle of attack using lagrangian coherent structures [8]

- A neural memristetor system with infinite or without equilibrium [9]

In the special issue, the analytical conditions for switchability conditions in discontinuous dynamical systems were presented. Based on the mapping structures, periodic motions in discontinuous dynamical systems are predicted with comparison of the numerical simulations. The materials presented herein give a comprehensive study of dynamics of discontinuous dynamical systems and shows the extensive applications. Based on the theory of discontinuous dynamical systems, the system synchronization mechanism was presented. Through numerical simulations, dynamics of the infinite equilibrium systems was presented. All materials in this special issue bring different points of view for discontinuous dynamical systems.

In the end, the editor would like to thank all authors for contributions and reviewers for providing help and service to keep high quality of manuscripts in this special issue. In addition, the editor would like to thank European Physical Journal-Special Topics, Sabine Lehr and Vincent Colpin for all supports. This special issue will benefit to our scientific community. I hope the materials will last very long for one to understand discontinuous dynamical systems and synchronizations.

\section{References}

1. J.Z. Huang, X.L. Fu, Eur. Phys. J. Special Topics 228, 1385 (2019)

2. J.Y. Chen, F.H. Min, Q.S. Jin, B.M. Ye, Eur. Phys. J. Special Topics 228, 1405 (2019)

3. X.L. Fu, S.S. ZHang, Eur. Phys. J. Special Topics 228, 1421 (2019)

4. Y.Y. Zhang, X.L. Fu, Eur. Phys. J. Special Topics 228, 1441 (2019)

5. A. Abedini, F.X. Wang, Eur. Phys. J. Special Topics 228, 1459 (2019)

6. S. Onsorynezhad, F.X. Wang, Eur. Phys. J. Special Topics 228, 1475 (2019)

7. H.Y. Ma, F.H. Min, Y.M. Lv, L. Zhang, Eur. Phys. J. Special Topics 228, 1493 (2019)

8. L. Wang, S.L. Cao, Y. Li, J.Z. Zhang, Eur. Phys. J. Special Topics 228, 1515 (2019)

9. F.Q. Wu, G. Zhang, J. Ma, Eur. Phys. J. Special Topics 228, 1527 (2019) 